\title{
Three crucial biological characteristics that's required for sustaining the livelihood of prokaryote and eukaryote cells
}

\section{Rishan Singh}

University of Kwa Zulu-Natal. Durban, 4001. South Africa. Email: rshnsingh1@yahoo.com.

\begin{abstract}
Since living organisms will always form an important part of the ecosystem, it's imperative to achieve a view on how imbalances cause problems. The one way in attaining this view is by understanding the way in which cells behave in different environments, ranging from the native environment to tested conditions. Cell viability, respiratory events as well as metabolic and nuclear shutdown are some critical events that occur, generally, in prokaryote and eukaryote cells. The distinct characteristics between the two cell types enable an advanced understanding about the occurrence of those features and processes attained during medicinal, and other cell-based studies.
\end{abstract}

Keywords: Cell metabolism; Prokaryote; Eukaryote; Evolution; Chromosomal aberration.

\section{Introduction}

Prokaryotes and eukaryotes, the two types of living cells on Earth, differ profoundly in cellularisation and the methods by which they differentiate and reproduce in nature (Vellai and Gábor, 1999). However, it's obvious, that both cell types produce waste products and utilise naturally-available food sources, thus making them known as living entities. The organisms were named as such because it was found that some of them were comprised of only one cell (prokaryotes), while others comprised of many different cell types (eukaryotes) (Vellai and Gábor, 1999). The differentiation between prokaryotes and eukaryotes, eventually, became more apparent when investigators become aware about the mechanisms by which both cell types reproduced in nature (Sogin, 1991). Eukaryote cells are far more complicated to study, because in mammals they are in a continuous state of growing, dying, multiplying, as well as recycling (Brown and Doolittle, 1997). However, eukaryote cells are as vast as prokaryote cells, thus, making both cell types interesting to perform studies on. The ideal method of culturing eukaryote and prokaryote cells differ greatly, though, the one thing we can be sure of, is they both can be maintained in tissue culture systems. These systems involve culture media that are normally preselected, based on the individual requirements that a cell needs to grow optimally in its natural environment (Martin-Piedra et al., 2004). This, in essence, means that eukaryote and prokaryote cells are in constant contact with 
selective pressures in the environment. Some of these pressures include natural conditions, like sun, rain, and wind. However, these pressures serve as a number of purposes, the one being that it allows cells to develop, as sort of, resistance, against unfavoured conditions during seasonal and climate variation (Holland, 1997). In addition, seasonal and climate variation have resulted in plants developing special structures and secreting substances that enable them to function optimally, particularly with respect to intracellular functions (Uphof, 1962). Now, many plant structures are not only studied for their medicinal properties, but also for their intra- and inter-cellular characteristics. These studies, usually, are histological in nature.

In the laboratory, however, plant and animal tissues are stained for determining the constituents they contain. These constituents may be either of medicinal, or other practical, importance (Uphof, 1962). However, it is also imperative to know that, metabolites present in prokaryote and eukaryote cells (cancer cells for example), when performing histological tests, may, in fact, represent the biological efficiency of the metabolic pathways of the cells being studied. This means that the constituents present in plant and eukaryote cells (mammalian cells) may, actually, cause a vast number of reactions in cells, and thus, the human body in the case of eukaryote cells. These reactions, in mammals, could cause disease states that are curable, detrimental, or have positive outcomes in the body. The positive outcomes in the body involve being able to overcome particular metabolic disorders. For example, in terms of the eukaryote cell cycle, many events, both positive and catastrophic, determine the fate of them in bodily functions. A chromosomal aberration for example, are dire catastrophic effects in mammals, sometimes causing mental deficits, and in some cases, phenotypic manifestations. In addition, there has also been immunological problems, due to eukaryote cell cycle interruptions, since all mammalian cells follow the same, if not similar, modes of death and recovery (or survival) (Woese and Fox, 1977). In unicellular organisms, signal transduction events do not occur, implying that the targeting of specific processes in eukaryote cells is, indeed, difficult. This means that the complexity of both cell types, would definitely, enable the infinite ravelling of new information for generations to come. In this proceedings article, the focus of discussion would be on cell viability, respiratory events, and metabolic and nuclear shutdown that has consequences for unicellular and multicellular growth, death and recovery strategies (health). Emphasis will be placed on differences between eukaryote and prokaryote cells, with the plant cells (to some degree) being central to both cell types.

\section{The concept of cell viability}

Cell viability, the inverse of cytotoxicity in mammalian tissue/cell culture, is a term used to refer to living cells. The term cell viability was probably discovered at the time when the HeLa (cervical cancer) cancer cell line was found to exhibit a very rapid proliferative potential. Cell viability and death are related terms, because cell death can only be obtained once the viable cell count has been recorded. In tissue culture experiments, cell death is calculated by subtracting the cell viable count value from one hundred. Although the calculation of cell death from cell viability is simple, the actual process involved to obtain the cell viable number is quite difficult.

The cell viability count is quite difficult to obtain, because of a series of subsequent cell culturing steps needed to be performed successfully first, so that the viable cell count obtained is a number that represents health cells only. However, cell viability depends on many factors, two of which are oxygen entry into cultured cell lines and performing proper decontaminating steps in the laboratory. This implies that cell viability can be affected by several factors, and that the contamination of cells by pathogens (like bacteria) and other in situ/in vitro debri, is perhaps a major factor that hinders the growth of healthy cells in the laboratory. The introduction of mitogens in tissue culture flasks is known to stimulate the viability of cells, however, there has been a few reports in which plant compounds and extracts have a cell proliferative effect (Majno and Joris, 1995; Vab Cruchten and Van Den Broeck, 2002; Lee et al., 2004; Goldtein and Kroemer, 2006). Obtaining the viability cell count (with, without, during, after or before treatment), with emphasis on mammalian cells such as 
fibroblasts, cancer cells, leukocytes, red blood cells etc., usually involves using the Trypan Blue method (Martin-Piedra et al., 2004). The method ensures that only viable cells are counted, but simultaneously allows for the investigation to view, unstained, dead cells in tissue culture flasks (Martin-Piedra et al., 2004). This method of obtaining the number of living (also dead) cells can become labourintensive, because sometimes the cells appear to be clumped when viewed under the haemocytometer. The clumping makes it distinct to obtain an accurate cell count. In the Trypan Blue method of cell counting, the viable cells appear blue (the colour of the stain) under the haemocytometer (Mossman, 1983). Cells that have been supplied with the adequate oxygen, incubation, temperature, and nutrient-rich media, would therefore appear bigger and thus healthier during regular microscopical investigations. Cell viability, therefore, also refers to cells that are respiring optimally - or less optimally - because in order for the cells to consume the Trypan Blue, they must be able to be metabolically active and, thus, respiring (Mossman, 1983). This metabolic activity in respiring cells is often seen during cytotoxicity tests when viable cells solubilise the formazan crystals using two enzymes as part of the oxidationreduction process. These enzymes would not be discussed or mentioned here, but what is important and must be highlighted, is that cell viability is an enzyme regulated feature that respiring cells exhibit under optimal laboratory conditions. These laboratory conditions are, and must always be, in line with the environment conditions of the host body. In order for cells to be viable, their cell membranes must be intact. In the case of plant cells, the plant cell wall enables the cells to be able to face harsh environment conditions. Therefore, the viability of plant cells are sustained in the environment due to the cell wall and other cellular features that vary from processes to secretions to structure (Uphof, 1962). In animal cells, maintaining a regular cell membrane is difficult because mammalian cells mobilise in the body, as is the case of red blood cells for example. Also, the process of cytoplasmic streaming makes the maintenance of the structure of mammalian cells difficult. The cell wall is, therefore, also a major physical barrier for chemical compounds that are able to promote cell growth because they need to first cross this outer layer of the cell before they are able to activate enzymes that are required for cell growth, division and multiplication (Rothman and Lenard, 1977; Singh, 2011). Cell viability thus depends on a genetically regulated programme involving cell cycle events and checkpoints that are regulated by enzymes (Lockshin and Zakeri, 2004). These enzymes include the cell cycle repressor P53, caspases, dehydrogenases, oxygenases, and many others (Majno and Joris, 1995; Van Cruchten and Van Den Broeck, 2002; Holdenrieder and Stieber, 2004; Lockshin and Zakeri, 2004; Golstein and Kroemer, 2007). This means that in order to understand cell viability, and thus death, one needs to know the genomics and proteomics involved in cell cycle events. This understanding, currently, indicates the reason behind the ability of substances having the capacities to enter and leave the cell, causing harm to the cell or leaving it unharmed. The machinery involved in enabling the entry and exiting of substances must, thus, involve some means by which those substances can cross the cell membrane. The one and only means by which that is made possible is through membrane embedded proteins or ligands (Rothman and Lenard, 1977 Woese and Fox, 1977). These proteins assist in the translocation of substances into and out of the cells, and thus are important for cell growth (Rothman and Lenard, 1977). Cells that receive an adequate supply of mitogens and nutrients grow through signal transduction events that are controlled by the nucleus, and assisted by protein synthesis at the ribosomes. Protein synthesis during cellular growth is, as it is known, mediated by messenger RNA (mRNA). Cell viability, therefore, involves transfer and ribosomal RNA molecules. In addition, it could be a complicated phase of a cells life to understand sue to the complexities of organisms that are available in nature. In tuberculosis research, for example, the level of tuberculosis cell viability is extrapolated relative to cell death in drug susceptibility tests (Singh, 2011). In HIV research, the latter method has also been used to measure cell viability (Singh, 2011).

\section{The respiration events}

Respiratory events, a sequence of mitochondrial regulated steps, refer to the 
events that are necessary to occur in order for cells to grow and survive (Chance and Williams, 1956; Rogers, 2005). These events, however, are dependent on holistic functioning of the organs, tissues and systems of the organism (Rogers, 2005). In bacterium, on the other hand, optimum respiration of the microorganism depends on the health state of the host in which they thrive (Singh, 2011). For example, certain microorganism species like Mycobacterium tuberculosis tend to prefer hosts in which the immune system is weakened (Singh, 2011). These microbes prefer weakened hosts, because they serve one primary purpose in nature, i.e. to harm, and eventually kill the host. Cancer and other mammalian cells, on the other hand, need aerobic respiration by the host in order for them to multiply in the body of the host optimally (Kondo and Kondo, 2006). In all mammalian cells, it is a reported fact that respiration is, indeed, an enzyme regulated process (Chance and Williams, 1956; Argaud et al., 1997). However, those respiratory events involve very difficult enzymes compared to the ones mentioned under the previous section on cell viability. Although this difference in the enzymes that participate in cell viability and respiration is marked, it is possible to generalise that the enzymes involved in cell death / viability are important for successful respiration of successively multiplying cells, particularly because without the cell viability enzymes, many if not all, cells would not be able to exist. This means that respiration events begin at the level of the organism, but depends on the optimum functioning of the respiratory chain of multicellular organisms they infect, affect, or manifest in (Chance and Williams, 1956). The same applies to microbes, fungi and other external infectants (that is, disease-causing pathogens) that may invade plants in the natural environment. In general, some plants have developed external structures over the last 4.6 billion years or evolution, enabling plants to be harmed minimally by external predators and microbes (Holland, 1997; Sogin, 1991). In plants, the respiratory events inferred to here are those events that occur in the chloroplast, i.e. the events that take place during photosynthesis (Rogers, 2005). These events involve either the presence or the absence of light, and are referred to as light respiration and dark respiration, respectively (Ordentlich et al., 1991). In mammalian cells the series of events that occur during respiration is complicated, because it involves many channeling molecules, and processes that are independent of one-and-other (Storey, 1971). These channeling molecules include electron acceptors, electron donors, phosphorylated molecules, and even high energy transferring carriers like nicotinimide diphosphate (NADH). In plant cells, NADH is called nicotinimide adenine dinucleotide phosphate, NADPH (Storey, 1971). The independent respiratory events in mammalian cells are oxidative phosphorylation, glucose breakdown (through a process called glycolysis) and waste dissipation through the Acetyl-CoA intermediate (Chance and Williams, 1956; Noguchi et al., 2013). Eukaryote cells, or mammalian cells as we know, require oxygen for respiration to take place (Woese and Fox, 1977; Sogin, 1991). However, it is equally essential that waste products, like pyruvate (which is released toward the end of glycolysis) is either used or destroyed in order to prevent cell death by necrosis. Necrosis, unlike apoptosis and autophagy, is a cell death mechanism that occurs in the absence of inducing or chemical substances (Lockshin and Zakeri, 2004; Kondo and Kondo, 2006). Therefore, it is for this reason, that, necrosis also refers to a form of cell death that occurs naturally (Majno and Joris, 1995; Van Cruchten and Van Den Broeck, 2002). However, all eukaryote cells, irrespective of whether they undergo necrosis, apoptosis, or autophagy, follow the same pathway of aerobic and oxidative respiration (Vellai and Gábor, 1999). In plant cells, respiring events that occur during photosynthesis involve a cyclic mechanism called the Calvin Benson Cycle (or Krebs Cycle). This cycle is essential to plants, because it enables plants in the natural, and laboratory environments, to dissipate oxygen and water as byproducts. As an obvious fact, this oxygen feeds into eukaryote cell aerobic respiration. On the far end, on the other hand, oxidative and anaerobic respiration mechanisms are distinct type of events that occur among specific microorganisms (Singh, 2011). These mechanisms had isolated 
anaerobic bacteria as a unique group of microorganisms, though they are equally important as aerobic and eubacteria in nature (Brown and Doolittle, 1997; Singh, 2011).

The respiration events, in some plants, are well adapted to the habitat they thrive in that they have evolved special adaptations to deal with their surroundings (Uphof, 1962). Seedweeds, for example, respire optimally in saline environments, because the stomata of underwater plants are adapted for water conservation. This means that hydrophytes are also, indeed, adapted to hydritic conditions, but less saline compared to the halophytes and xerophytes. In all three plant types, the respiratory events probably overlap, but only to a certain extent because of climate variations and them possessing adaptations for water loss and retention. Eukaryote cells are surrounded by a membrane, while plants have an outermost later known as the cell wall (as mentioned) (Vellai and Gábor, 1999). In order to arrest respiration events in cells, receptors spanning the outermost layer in plant and animal cells, first need to be activated in order to mediate cell cycle arrest (Roger, 2005; Reape et al., 2008). When a compound, or toxin, reaches the outermost layers of the cell, the respiration rate declines because the intermediates of oxidative phosphorylation, for example, in eukaryote cells, begin to receive an inadequate shuffle of electrons and protons, resulting in the build-up of toxins within the cell (Chance and Williams, 1956). Furthermore, the adenosine diphosphate molecules are unable to become completely phosphorylated. This results in cell cycle inhibition, which eventually leads to cell cycle arrest and cell death. In addition, and depending on the cell cycle event being affected by cell inducing or chemical substances, certain proteins involved in regulating the level, or extent, of cell viability may become activated (Majno and Joris, 1995; Van Cruchten and Van Den Broeck, 2002; Kondo and Kondo, 2006). Caspases are an example of one type of protease that participate in executing cell death in the mitochondria (Majno and Joris, 1995; Van Cruchten and Van Den Broeck, 2002; Holdenrieder and Stieber, 2004; Kondo and Kondo, 2006). However, this mode of cell death can be initiated by membrane proteins, or ligands, as an independent mode of cell death compared to the mitochondrial mode of cell death (Van Cruchten and Van Den Broeck, 2002). Another mode of eukaryote cell death is via the endoplasmic reticulum, but this mode isn't popularly reported in the literature (Van Cruchten and Van Den Broeck, 2002). In plants, rubisco, an important enzyme involved in maintaining the oxygen/carbon dioxide ration, is one of the main proteins that regulate respiration in nature. Rubisco is important in carboxylation reactions involving oxygen in plants. The respiration events in plants, therefore, include the participation of grana, the porous thylakoid membrane as well as both photosystems, because there could be considered major requirements for sustaining the ecosystem through changes in climate and seasons (read section on rubisco in Salisbury and Ross, 2000). Furthermore, the sodium and potassium channels spanning the thylakoid membranes, in-turn, regulate the exchange of gases during photorespiration (herein referred to as photosynthesis) in the light phase, more prominently (Salisbury and Ross, 2000). In prokaryotes, respiration is controlled by proteins as is the situation with tuberculosis (Singh, 2011). HIV growth is also controlled by proteins (Singh, 2011). However, the proteins ensure that rapid multiplication of the virus is maintained.

\section{Metabolic and nuclear shutdown}

Metabolic and nuclear shutdown, the two processes that occur when cell death is activated. are important steps that take place when cells, such as red blood cells for example, have reached the end of their lifespan and thus need to be eliminated from the body. The processes are, indeed, controlled by enzymes (Georgatos et al., 1997; Wolf and Green, 1999). However, although a decline in the metabolic activities in plant and animal cells are related to the events that occur at the nucleus when death is activated, they could be independent events because the metabolic pathways in eukaryote (mammal) and plant cells are catalysed by different enzymes. Metabolic shutdown refers to the stopping of the events that involve the 
synthesis and breakdown, or hydrolysis, of the precursor molecules that are required for optimal functioning of tissue organ systems, and thus the human body. Nuclear shutdown, on the other hand, refers to when the nucleus no longer regulates, or controls, cellular functions optimally (Georgatos et al., 1997). The overall features of metabolic and nuclear breakdown, are, however, distinct from each other. In metabolic shutdown, the process of glycolysis for example may be interrupted, thereby causing excessive amounts of saccharides to accumulate in eukaryote cells (Noguchi et al., 2013). This means that metabolic shutdown, indeed, does have vast consequences that people could encounter if metabolic shutdown does occur (Noguchi et al., 2013). One of these consequences is the development of diabetes, due to a deficient glycolytic pathway (Bruce et al., 1988; Aiston et al., 2003; Noguchi et al., 2013). Other pathways that could be deficient, resulting in metabolic shutdown due to the accumulation of unused harmful and harmless precursor and end product molecules, is the nitrogen cycle, amino acid cycle as well as gluconeogenesis in mammalian cells. An interruption in any one of the mentioned cycles in mammals, thus, may lead to metabolic disorders such as anorexia nervosa, bulimia, kwashiorkor, as well as mental, or psychological, problems such as anxiety and depression, eventually leading to the people who suffer from metabolic shutdown to die. Since metabolic shutdown results in the accumulation of waste substances in the body, death usually occurs when systems are unable to cope with those substances e.g. lactic acid, ammonia and pyruvate accumulation. It's evident from everything already mentioned that mammalians must have strategies to overcome their bodies from undergoing metabolic shutdown. Cell viability is an inherent feature which enables cells to grow on a continuous basis in healthy persons, thereby affording the bodies of mammals a type of 'detoxification' strategy so that the systems can function optimally. This strategy, thus, enables the systems of the body to be in a state of homeostasis. Homeostasis, by definition, is a process in which the biological processes that occur in the bodies of mammals are in a state of balance. This balance occurs mostly because two substances or compounds, that have effects that oppose each other in eukaryote cell systems, are in equal concentration to each other in the body. Any imbalance in the concentration of the opposing substances may result in side effects experienced by mammals. For example, an imbalance between pancreatic lipases and pancreatic pepsin may cause metabolic imbalance side effects in mammals. These effects include an imbalance in blood lipid, (or cholesterol), and proteins, concentrations because the lipids or proteins that enter the pancreas aren't adequately hydrolysed into smaller molecules for optimal serum lipid and protein concentrations to be reached. This means that metallic shutdown, evidently, does occur at the organ level in mammals (Bruce et al., 1988; Aiston et al 2003). In plants, on the other hand, metabolic shutdown occurs when an interruption in the photosynthetic pathways, i.e. like photosystems I and photosystems II, result in oxygen accumulation within the leaves (Rogers, 2005, although not related to this statement). This, in-turn, causes the oxidation of all molecules within the leaves, eventually leading to death (Rogers, 2005, although not related to this statement). In both plant and eukaryote (mammalian) cells, metabolic breakdown instigates nuclear breakdown, however, the inverse situation is, most definitely, true as well. Nuclear shutdown causes the cell to receive either conflicting signals, or no signal at all, resulting in plant and animal cells to develop the ability to lose their structure, integrity and function (Vellai and Gábor, 1999). In eukaryote (mammalian) cells, nuclear shutdown also occurs when the nucleus becomes fragmented when the cell encounters a toxin (Majno and Joris, 1995; Van Cruchten and Van Den Broeck, 2002; Kondo and Kondo, 2006). In the laboratories, nuclear shutdown is measured by obtaining an estimate about the quantity of toxin that has entered the cytoplasm of the cell. Obtaining the estimate isn't always easy and can involve using many methods (Mossman, 1983). Although these methods will not be mentioned here, they are all well-established and involve a colour change of some kind or the other (Mossman, 1983). Nuclear 
shutdown in eukaryote (mammalian) cells, certainly, cause the collapse of the cytoskeleton elements indirectly if shutdown causes death after membrane integrity has become altered by chemical compounds, including those that cause DNA damage (Rothman and Lenard, 1977; Georgatos et al., 1997).

In prokaryotes, like disease-causing bacterium species, metabolic and nuclear shutdown occurs when cells encounter effective treatment modalities. These modalities may be in the form of capsules, tablet, syrups and intravenous injections. However, some stains of TB, for example, have become accustomed to treatment regimens in some hosts, resulting in failure to cause metabolic and nuclear shutdown (Singh, 2011). This failure is related to the fact that such strains are, most definitely, resistant to treatment regimens (Singh, 2011). The prokaryotes are distinct in their own, compared to eukaryotes, because they possess a nucleoid, and not a nucleus like in most (if not all) multicellular organisms (Vellai and Gábor, 1999). This means that nuclear shutdown could, most probably, be induced more easily compared to eukaryotes because the nucleoid is a more primitive structure compared to the nucleus in eukaryotes, in which it is a membrane-bound organelle (Vellai and Gábor, 1999). Furthermore, it is the absence of a membrane-bound nucleus in prokaryotes that enable the quick hindrance of prokaryote cell growth, for example in the amoeba. Overall, from the given discussion, it is clear that metabolic and nuclear shutdown cannot be separated effectively because a variety of mechanisms involved in metabolic regulation in mammals, and it is the nucleus that maintains function at the multicellular level. In viruses, like HIV, metabolic and nuclear shutdown has been reported, but details will not be provided in this article.

\section{Conclusion}

It is evident that prokaryote and eukaryote cells are unique. However, much more research into understanding the physiological responses in eukaryotes must be achieved because physiological responses, are an obvious trait, exhibited by all multicellular organisms. This means that, although the prokaryotes are well established organisms on Earth, physiological responses, and thus metabolites, is through primitive organelles suspended in the cytoplasm of the cell. Also certain traits, one being vacuolisation in the prokaryotes, is an established feature of primitive living organisms (Vellai and Gábor, 1999). In contrast, metabolism in eukaryote cells can by means of digestion take place in membrane-surrounded vacuoles, this includes all plants including many underwater plants and xerophytes. Proper respiratory mechanisms, for cell growth, in fauna and flora, are in place since after evolution occurred. Although respiration is a trait that enables cells to survive in nature, without healthy cells, and a proper cell viability cycle, cell respiration wouldn't have a drastic effect on cellular organisms. In the mammalian body, often, respiration is reported at the organ level, however, here, it has been reported at the cellular level of organisms. However, since cells comprise the system of mammals, it is, or could be considered, best to study the different biological parameters at the level of the cell so that a microscopy-like view on occurrences at the tissue level, and organ level can be achieved with greater accuracy. In this article, the focus was on cell viability, respiration events, and metabolic and nuclear shutdown, as they have been considered important for all living organisms. Although it provides a glimpse into some features and occurrences in biology, the parameters discussed, here, have consequences for all of civilization.

\section{Conflicts of interest}

Author declares that they have no conflict of interests.

\section{References}

Aiston, S.; Andersen, B.; Agius, L. Glucose-6phosphate regulates hepatic glycogenolysis through inactivation of phosphorylase. Diabetes, v. 52, p. 1333-1339, 2003. Available from: $<$ http://diabetes.diabetesjournals.org/content/diabe tes/52/6/1333.full.pdf $>$. Accessed on: April 23, 2017. 
Argaud, D.; Kirby, T. L., Newgard, C. B.; Lange, A. J. Stimulation of glucose-6-phosphatase gene expression by glucose and fructose-2,6bisphosphate. Journal of Biological Chemistry, $\begin{array}{lll}\text { v. 272, } & \text { p. 12854-12861, }\end{array}$ https://doi.org/10.1074/jbc.272.19.12854

Brown, J. R.; Doolittle, W. F. Archae and the prokaryote-to-eukaryote transition. Microbiology and Molecular Biology Reviews, v. 61, no. 4, p. 456-502, 1997. Available from: <https://www.ncbi.nlm.nih.gov/pmc/articles/PMC 232621/pdf/610456.pdf $>$. Accessed on: April 23, 2017.

Bruce, D. G.; Chisholm, D. J.; Storlien, L. H.; Kraegen, E. W. Physiological importance of deficiency in early prandial insulin secretion in non-insulin-dependent diabetes. Diabetes, v. 37, no. 6, p. 736-744, 1988. https://doi.org/10.2337/ diab.37.6.736

Chance, B.; Williams, G. R. The respiratory chain and oxidative phosphorylation. In: Nord, F. F. Advances in Enzymology and Related Areas of Molecular Biology. London: Wiley, 1956. v. 17 , p. $65-134$. https://doi.org/10.1002/ 9780470122624.ch2

Georgatos, S. D.; Pyrpasopoulou, A.; Theodoropoulos, P. A. Nuclear envelope breakdown in mammalian cells involves stepwise lamina disassembly and microtubule-driven deformation of the nuclear membrane. Journal of Cell Science, v. 110, p. 2129-2140, 1997. Available from: < https://pdfs.semanticscholar.org/ 8f7a/1bdf526ca1353f119855c1669773379f71cc.p df $>$. Accessed on: April 23, 2017.

Golstein, P.; Kroemer, G. Cell death by necrosis: towards a molecular definition. Trends in Biochemical Sciences, v. 32, no. 1, p. 37-43, 2007. https://doi.org/10.1016/j.tibs.2006.11.001

Holdenrieder, S.; Stieber, P. Apoptotic markers in cancer. Clinical Biochemistry, v. 37, no. 7, p. 605-617, 2004. https://doi.org/10.1016/ j.clinbiochem.2004.05.003

Holland, H. D. Evidence for life on Earth more than 3850 million years ago. Science, v. 275, no. 5296, p 38-39, 1997. https://doi.org/10.1126/ science.275.5296.38

Kondo, T.; Kondo, S. Autophagy and cancer therapy. Autophagy, v. 2, p. 85-90, 2006. https://doi.org/10.4161/auto.2.2.2463

Lee, J. Y.; Hwang, W. I.; Lima, S. T. Antioxidant and anticancer activities of organic extracts from Platycodon grandiflorum A. De Candolle roots. Journal of Ethnopharmacology, v. 93, p. 409-415, 2004. https://doi.org/10.1016/j.jep. 2004.04.017
Lockshin, R. A.; Zakeri, Z. Apoptosis, autophagy, and more. The International Journal of Biochemistry \& Cellular Biology, v. 36, no. 12 , p. 2405-2419, 2004. https://doi.org/ 10.1016/j.biocel.2004.04.011

Majno, G.; Joris, I. Apoptosis, oncosis and necrosis: an overview of cell death. American Journal of Pathology, v. 146, p. 3-15, 1995. Available from: <https:/www.ncbi.nlm.nih.gov/ pmc/articles/PMC1870771/pdf/amjpathol000490010.pdf>. Accessed on: April 23, 2017.

Martin-Piedra, M. A.; Garzon, I.; Celeste Oliveira, A.; Alfonso-Rodriquez, C. A.; Carriel, V.; Scionti, G.; Alaminos, M. Cell viability and proliferation capability of long-term human dental pulp stem cell cultures. Cytotherapy, v.16, p. 266-277, 2004 http://dx.doi.org/10.1016/j.jcyt.2013.10.016

Mossman, T. Rapid colorimetric assay for cellular growth and survival. Application to proliferation and cytotoxicity assay. Journal of Immunological Methodology, v. 65, no. 1/2, p. 55-63, 1983. https://doi.org/10.1016/00221759(83)90303-4

Noguchi, R.; Kubota, H.; Yugi, K.; Toyoshima, T.; Komori, Y.; Soga, T.; Kuroda, S. The selective control of glycolysis, gluconeogenesis and glycogenesis by temporal insulin patterns. Molecular Systematics and Biology, v. 9, 664, 2013. https://doi.org/10.1038/msb.2013.19

Ordentlich, A.; Linzer, R. A.; Raskin, I. Alternative respiration and heat evolution in plants. Plant Physiology, v. 97, p. 1545-1550, 1991. https://doi.org/10.1104/pp.97.4.1545

Reape, T. J.; Molony, E. M.; McCabe, P. F. Programmed cell death in plants: distinguishing between different modes. Journal of Experimental Botany, v. 59, no. 3, p. 435-444, 2008. https://doi.org/10.1093/jxb/erm258

Rogers, H. J. Cell death and organ development in plants. Current Topics in Developmental Biology, v. 71, p. 225-261, 2005. https://doi.org/ 10.1016/S0070-2153(05)71007-3

Rothman, T .E.; Lenard, J. Membrane asymmetry. Science, v. 195, no. 4280, p. 743-753, 1977. https://doi.org/10.1126/science.402030

Salisbury, B. F.; Ross, W. C. Plant physiology. 5. ed. Belmont: Wadsworth Publ. Co., 2000.

Singh, R. Mycobacterium tuberculosis: telling a story of truths. Journal of Pure and Applied Microbiology, v. 5, no. 2, p. 541-544, 2011.

Sogin, M. L. Early evolution and the origin of eukaryotes. Current Opinion in Genetics and Development, v. 1, no. 4, p. 457-463, 1991. https://doi.org/10.1016/S0959-437X(05)80192-3 
Storey, B. T. The respiratory chain of plant mitochondria. XI. Electron transport from succinate to endogenous pyridine nucleotide in mung bean mitochondria. Plant Physiology, v. 48, no. 6, p. 694-701, 1971. https://doi.org/ 10.1104/pp.48.6.694

Uphof, J. L. Y. Plant hair. Encyclopedia of Plant Anatomy, v. 4, no. 5, p. 1-206, 1962.

Van Cruchten, S.; Van Den Broeck, W. Morphological and biochemical aspects of apoptosis, oncosis and necrosis. Anatomy, Histology and Embryology, v. 31, no. 4, p. 214-223, 2002. https://doi.org/10.1046/j.14390264.2002.00398.x

Vellai, T.; Gábor, V. The origin of eukaryotes: the difference between prokaryotic and eukaryotic cells. Proceeding of the Royal Society of
London B, v. 266, no. 1428, p. 1571-1577, 1999. https://doi.org/10.1098/rspb.1999.0817

Woese, C. R. Bacterial evolution. Microbiological Reviews, v. 51, no. 2, p. 221-271, $1987 . \quad$ Available from: <https://www.ncbi.nlm.nih.gov/pmc/articles/PMC 373105/pdf/microrev00049-0051.pdf $>$. Accessed on: April 23, 2017.

Woese, C. R.; Fox, G.E. The concept of cellular evolution. Journal of Molecular Evolution, v. 10, no. 1, p. 1-6, 1977. https://doi.org/10.1007/ BF01796132

Wolf, B. B.; Green, D. R. Suicidal tendencies: apoptotic cell death by caspase family proteinases. Journal of Biological Chemistry, v. 274, p. 200049-20052, 1999. https://doi.org/10.1074/ jbc.274.29.20049 